\title{
Preparation and Characterization of
}

\section{a Collagen-Liposome-Chondroitin Sulfate Matrix with Potential Application for Inflammatory Disorders Treatment}

\author{
Oana Craciunescu, ${ }^{1}$ Alexandra Gaspar, ${ }^{1}$ Mihaela Trif, ${ }^{2}$ Magdalena Moisei, ${ }^{2}$ \\ Anca Oancea, ${ }^{1}$ Lucia Moldovan, ${ }^{1}$ and Otilia Zarnescu ${ }^{3}$ \\ ${ }^{1}$ National Institute of Research and Development for Biological Sciences, No. 296, Splaiul Independentei, Sector 6, \\ 060031 Bucharest, Romania \\ ${ }^{2}$ Institute of Biochemistry of the Romanian Academy, No. 296, Splaiul Independentei, Sector 6, 060031 Bucharest, Romania \\ ${ }^{3}$ Faculty of Biology, University of Bucharest, No. 91-95, Splaiul Independentei, Sector 5, 050095 Bucharest, Romania
}

Correspondence should be addressed to Lucia Moldovan; moldovanlc@yahoo.com

Received 19 March 2014; Accepted 28 April 2014; Published 18 May 2014

Academic Editor: Chunyi Zhi

Copyright (c) 2014 Oana Craciunescu et al. This is an open access article distributed under the Creative Commons Attribution License, which permits unrestricted use, distribution, and reproduction in any medium, provided the original work is properly cited.

\begin{abstract}
Smart drug delivery systems with controllable properties play an important role in targeted therapy and tissue regeneration. The aim of our study was the preparation and in vitro evaluation of a collagen (Col) matrix embedding a liposomal formulation of chondroitin sulfate (L-CS) for the treatment of inflammatory disorders. Structural studies using Oil Red O specific staining for lipids and scanning electron microscopy showed an alveolar network of nanosized Col fibrils decorated with deposits of L-CS at both periphery and inner of the matrix. The porosity and density of Col-L-CS matrix were similar to those of Col matrix, while its mean pore size and biodegradability had significantly higher and lower values $(P<0.05)$, respectively. In vitro cytotoxicity assays showed that the matrix system induced high cell viability and stimulated cell metabolism in L929 fibroblast cell culture. Light and electron micrographs of the cell-matrix construct showed that cells clustered into the porous structure at $72 \mathrm{~h}$ of cultivation. In vitro diffusion test indicated that the quantity of released CS was significantly lower $(P<0.05)$ after embedment of L-CS within Col matrix. All these results indicated that the biocompatible and biodegradable Col-L-CS matrix might be a promising delivery system for local treatment of inflamed site.
\end{abstract}

\section{Introduction}

Drug delivery systems (DDS) are designed for targeting the injured site with increased specificity and selectivity, for local efficient release of high drug concentration and, some of them, for initiation of tissue regeneration [1]. Liposomes (L) are drug delivery vehicles used in 13 medical products on the market; more than 25 liposomal drug formulations are currently in clinical trials [2]. Their successful application in treatment protocols depends on $\mathrm{L}$ composition and physical properties, including size, dispersity, morphology, and surface charge. Several studies have demonstrated L ability to protect drug from biological environment, to enhance drug intracellular uptake, and to modulate drug pharmacokinetics, biodistribution, and controlled release, thus representing a superior mode of drug administration compared to conventional forms [3]. In particular, for articular cartilage treatment, it was shown that empty L prepared from natural phospholipids improved joint lubrication and prevented degenerative changes [4]. Biologic active substances entrapped in L intra-articularly administered in inflamed joints targeted the phagocytic cells from synovium, suppressed proinflammatory cytokines, prolonged their residence time in the joint, and had decreased side effects [5]. Our previous studies showed that negatively charged L entrapping lactoferrin prolonged the anti-inflammatory effect in DBA1 mice with collagen-induced arthritis, compared to free lactoferrin [6]. 
The versatility, attractive biologic properties, and higher uptake of drug liposomal formulations are benefits that recently boosted their use instead of drug alone, but problems related to L permeability were reported [7]. A new generation of smart DDS combines the controlled release of drug encapsulated in nanocarriers with the structural advantages of $3 \mathrm{D}$ polymeric networks, for enhancing lipid bilayer stability and drug efficiency. Hydrogel matrices were designed in various compositions, from natural and synthetic polymers, as carrier systems for intra-articular treatment of joint disorders. They provided several advantages compared to other colloidal DDS, such as control of drug release, a suitable microenvironment for cell proliferation, and initiation of new cartilage matrix synthesis [8]. The main limitation of hydrogels was lack of mechanical strength due to random alignment of fibres and high content of water [9]. Polymeric electrospun mats, analyzed as delivery systems of nanoparticles, hindered cell infiltration due to pore diameter size smaller than $10 \mu \mathrm{m}$ [10]. Carbon nanotubes were used in drug delivery applications to take advantage of their high conductivity and stability, but a key problem was their biosafety [11]. Therefore, composites of stimuli-responsive polymers and carbon nanotubes were fabricated, and they showed a good cell response [12]. Freeze-dried porous scaffolds have ideal attributes as depots for drug-loaded nanocarriers, presenting controllable mechanical properties, biomimetic structure that favored cell adhesion, and control of drug delivery [13].

It is known that collagen $(\mathrm{Col})$ is a natural, biodegradable polymer with low immunogenicity. Several composites based on Col were used both as DDS for bioactive molecules and scaffolds that guide cell adhesion, proliferation, and differentiation to stimulate new extracellular matrix components synthesis during regenerative processes [14, 15]. Chondroitin sulfate (CS), the main glycosaminoglycan (GAG) of articular cartilage extracellular matrix, is currently used as a chondroprotective agent in clinical studies. In vitro and in vivo reports have showed CS anti-inflammatory activity and, as a result, its role in reducing several parameters of synovitis, including cell infiltration, fibrosis, and proliferation of synovial lining cells [16]. We previously showed that a liposomal formulation of CS protected fibroblast cells from inflammation process [17].

The present study aimed to prepare a DDS consisting of CS encapsulated in L (L-CS) embedded in a natural polymeric matrix of Col (Col-L-CS) for local treatment of inflammatory and degenerative disorders, specifically osteoarthritis (OA). Col-L-CS was structurally and physicochemically characterized. Its in vitro cytotoxicity was evaluated in two models of direct and indirect contact with L929 mouse fibroblast cells. CS release was assessed spectrophotometrically at different periods of time.

\section{Materials and Methods}

2.1. Materials. CS-A sodium salt from bovine trachea, L$\alpha$-phosphatidylcholine type XVI-E from egg yolk (PC), dioleoyl-phosphatidyl-ethanolamine (DOPE), stearylamine
(SA), cholesterol (Chol), Triton X-100, bacterial collagenase type IA (E.C. 3.4.24.3), Minimum Essential Medium (MEM), fetal calf serum (FCS), L-glutamine, trypsin (E.C. 3.4.21.4), ethylenediaminetetraacetic acid (EDTA), 3-(4,5dimethylthiazol-2-yl)-2,5-diphenyltetrazolium bromide (MT $\mathrm{T}$ ), and all other chemicals and solvents of analytical grade were purchased from Sigma-Aldrich Chemicals (Germany). Sephadex G-25 column was purchased from Sigma-Aldrich Chemicals (Germany). Blyscan kit was purchased from Biocolor Ltd. (Northern Ireland, UK). A mouse fibroblast cell line (NCTC clone L929) was purchased from ECACC (Sigma-Aldrich, Germany).

2.2. Preparation and Characterization of $L-C S$. Lipid vesicles entrapping CS were prepared using the thin film hydration method, as previously described [18]. Briefly, a lipid phase (14 mg total lipids $/ \mathrm{mL}$ ) was prepared from a mixture of lipids PC:DOPE:Chol:SA, in 4:2:3:1 molar ratio, and dissolved in a solution of chloroform/methanol $(95: 5, \mathrm{v} / \mathrm{v})$. The solvents were removed in an IKA RV 10 rotary evaporator (Ika-Works Inc., Germany), at $150 \mathrm{rpm}, 40^{\circ} \mathrm{C}$, for $30 \mathrm{~min}$, under reduced pressure. The resulted thin, dry lipid film was hydrated in PBS, pH 7.4, containing $10 \mathrm{mg} / \mathrm{mL}$ CS, and L-CS suspension was incubated at room temperature, for $5 \mathrm{~h}$, to allow complete swelling of the lipid film. In order to obtain a homogeneous population, the liposomal suspension was sonicated under inert atmosphere of $\mathrm{N}_{2}$ first in a bath-type sonicator (Grant, $\mathrm{UK}$ ), at $37^{\circ} \mathrm{C}$, for $2 \times 30 \mathrm{~min}$ and then in a probe-type sonicator (Bandelin Sonopuls, Germany), at $70 \%$ amplitude, for $3 \times 10 \mathrm{~s}$. L-CS were separated from nonencapsulated CS by size exclusion chromatography using a Sephadex G-25 column and stored at $4^{\circ} \mathrm{C}$ until use. For cell culture experiments, L-CS were sterile filtered through $0.2 \mu \mathrm{m}$ filters.

The size and polydispersity index (PI) of L-CS population was determined by dynamic light scattering (DLS) technique using a ZetaPlus instrument (Brookhaven Instruments Corporation, Holtsville, NY) operated at $658 \mathrm{~nm}, 90^{\circ}$ angle, at $25^{\circ} \mathrm{C}$, as previously described [19].

The encapsulation efficiency (EE) was assessed by CS quantification using Blyscan GAG kit after L-CS solubilization in $0.1 \%$ Triton X-100, as previously described [20]. The optical density (OD) of the colored solution was measured at a wavelength of $656 \mathrm{~nm}$, in a Sunrise microplate reader (Tecan, Austria). Entrapped CS concentration was determined on the calibration curve in the range $0-5 \mu \mathrm{g}$ GAG. EE was calculated as percentage from total CS using the following equation:

$$
\mathrm{EE}(\%)=\frac{\text { entrapped CS }}{\text { total CS }} \times 100 \% .
$$

The results were expressed as mean of three independent determinations \pm standard deviation (SD).

2.3. Preparation of Col-L-CS Matrix. A solution of Col type I was prepared from bovine tendons by pepsin treatment, in a dry tissue: enzyme ratio of $1: 10(\mathrm{w} / \mathrm{w})$. Then, the solution was purified by precipitation at a salt concentration of $2.4 \mathrm{M}$ 
$\mathrm{NaCl}$ and dialyzed against distilled water to remove salt, as previously described [21]. Col type I gel $(0.75 \%$, w/w) was mixed with L-CS suspension, in a ratio of $1: 1(\mathrm{w} / \mathrm{w})$, using a manual speed-stirrer (Xenox, Germany), at 1,000 rpm. The mixture was poured in glass molds ( $20 \mathrm{~mm}$ diameter), put on the shelf of a Christ freeze-dryer (Germany), and frozen to $-35^{\circ} \mathrm{C}$. The ice crystals formed during freezing were removed by a drying process using a step program consisting of $0^{\circ} \mathrm{C}$ stage, at $0.26 \mathrm{mbar}$, for $17 \mathrm{~h}$ and $30^{\circ} \mathrm{C}$ stage, for $10 \mathrm{~h}$, as previously described [22]. This controlled process resulted in pore formation in the $3 \mathrm{D} \mathrm{Col}$ sponge matrix. The samples of Col-L-CS matrix were sealed in plastic bags and irradiated by UV exposure in a Scie-Plas cabinet (UK), for $8 \mathrm{~h}$, to sterilize and strengthen them. A Col matrix was prepared in the same conditions for control.

2.4. Structural Characterization. Histological examination of Col-L-CS matrix was performed after fixation in Ciaccio solution, dehydration in ethanol, clarifying in toluene, and embedding in paraffin. Sections of $5 \mu \mathrm{m}$ were stained with $0.1 \%(w / w)$ Oil Red O, mounted in glycerol, and observed at an AxioCam MRc5 microscope equipped with camera driven by AxioVision 4.6 software (Carl Zeiss, Germany).

The porous matrix ultrastructure was examined by SEM. Matrix samples were examined at surface and cross-sections cut with a razor blade, mounted on carbon pads attached to aluminium stubs, and visualized in the low vacuum mode, $25 \mathrm{kV}$, at a FEI Quanta 400 Environmental Scanning Electron Microscope (ESEM, Philips, The Netherlands). The pore size was calculated by image analysis on at least 100 pores for each sample using ImageJ software.

\subsection{Physicochemical Characterization}

2.5.1. Porosity and Density. Matrix porosity $(\varepsilon)$ and density (d) were measured by the water displacement method, as previously described [23]. Briefly, a sample with a known weight $(w)$ was immersed in a graded test tube holding a known volume of water $\left(v_{1}\right)$. The sample was kept in water for $3 \mathrm{~h}$ and pressed to force air from the matrix and allow water to penetrate and fill the pores. The total volume of water plus the water-impregnated matrix was recorded as $v_{2}$. The water-impregnated matrix was removed from the test tube and the residual water volume was recorded as $v_{3}$. The following equations were used:

$$
\begin{aligned}
& \varepsilon=\frac{\left(v_{1}-v_{3}\right)}{\left(v_{2}-v_{3}\right)} \times 100, \\
& d=\frac{w}{\left(v_{2}-v_{3}\right)} \times 100 .
\end{aligned}
$$

The results were reported as mean of three measurements $\pm \mathrm{SD}$.

2.5.2. Biodegradability Tests. For in vitro degradation measurement, a sample of Col-L-CS matrix was weighed ( $10 \mathrm{mg})$ and was preincubated in $5 \mathrm{~mL}$ TES buffer, $\mathrm{pH} 7.4$, containing $50 \mathrm{mM} \mathrm{CaCl}_{2}$, at $37^{\circ} \mathrm{C}$, for $30 \mathrm{~min}$. Then, $100 \mu \mathrm{L}$ TES buffer containing or not $100 \mu \mathrm{g} / \mathrm{mL}$ collagenase type IA was added and the degradation took place in a water bath, at $37^{\circ} \mathrm{C}$, for $6 \mathrm{~h}$. The reaction was stopped by addition of $0.2 \mathrm{~mL} 0.25 \mathrm{M}$ EDTA and cooling on ice. After centrifugation at 6,000 rpm, for $10 \mathrm{~min}, 200 \mu \mathrm{L}$ of supernatant was analyzed for amino acids content by ninhydrin method, as previously described [24]. The results were calculated as percentage from the completely degraded matrix (100\%) and were reported as mean of three independent experiments \pm SD. A Col matrix processed in the same conditions served as control.

\subsection{In Vitro Cytotoxicity Tests}

2.6.1. Cell Culture. Mouse fibroblasts from NCTC clone L929 cell line were grown in T75 flasks in MEM supplemented with $10 \%$ FCS, 2 mM L-glutamine, and 1\% mixture of antibiotics. The culture was maintained in an incubator with humidified atmosphere of $5 \% \mathrm{CO}_{2}$ and $95 \%$ air, at $37^{\circ} \mathrm{C}$. For all experiments, cells were harvested from subconfluent cultures using $0.25 \%$ trypsin-EDTA solution and were resuspended in fresh serum supplemented MEM before plating.

2.6.2. Indirect Cytotoxicity Test. Cell suspension was seeded at a density of $5 \times 10^{4}$ cells/well, in 24-well culture plates, and plates were incubated in $5 \% \mathrm{CO}_{2}$ humidified atmosphere, at $37^{\circ} \mathrm{C}$, for $18 \mathrm{~h}$, to allow cell adhesion. Then, sterile samples of Col-L-CS matrix and L-CS suspension were added in the culture medium to give a final CS concentration of $0.5 \mathrm{mg} / \mathrm{mL}$. Plates were incubated at $37^{\circ} \mathrm{C}$, in standard conditions of cultivation, for $24 \mathrm{~h}, 48 \mathrm{~h}$, and $72 \mathrm{~h}$, respectively. Cell viability was assessed by MTT assay, and cell morphology was observed by light microscopy, as described below.

The effect of possible toxic compounds released from the samples on the mitochondrial succinate dehydrogenases activity was evaluated using MTT assay, as previously described [25]. Briefly, the culture medium was replaced with fresh medium containing MTT solution, in a $10: 1$ (v/v) ratio, after each period of incubation, and the plates were incubated at $37^{\circ} \mathrm{C}$, for $3 \mathrm{~h}$. Then, $500 \mu \mathrm{L}$ of isopropanol was added to each well to dissolve the formazan crystals by gently being shaken on a platform, for $3 \mathrm{~h}$. The colored solution was transferred to a 96-well plate and the OD was read at $570 \mathrm{~nm}$, using a Sunrise microplate reader (Tecan, Austria). The measured OD is directly proportional to cell viability and the results were calculated using the following equation:

$$
\text { Cell viability }(\%)=\frac{\mathrm{OD}_{\text {sample }}}{\mathrm{OD}_{\text {negative control }}} \times 100 \text {. }
$$

The cells cultured in complete culture medium served as negative control, while cells treated with $50 \mu \mathrm{M}$ hydrogen peroxide served as positive control. A Col matrix and CS solution were also used as controls. Three separate experiments were conducted and the results were expressed as mean $\pm \mathrm{SD}$.

For cell morphology observations, the medium was discarded after $72 \mathrm{~h}$ of cultivation and the cells were fixed in methanol and stained with hematoxylin-eosin (H\&E). The 
cultures were photographed at a Zeiss AxioStar Plus microscope equipped with a digital camera driven by AxioVision 4.6 software (Carl Zeiss, Germany).

2.6.3. Direct Cytotoxicity Test. Sterile samples of Col-L-CS matrix $\left(0.5 \times 0.5 \times 0.5 \mathrm{~cm}^{3}\right)$ were placed in the wells of a 24-well culture plate. L929 mouse fibroblast cell suspension $(200 \mu \mathrm{L})$ containing $1 \times 10^{6}$ cells was injected into each sample, followed by incubation for $2 \mathrm{~h}$. Then, $500 \mu \mathrm{L}$ MEM supplemented with $10 \%$ FCS was added into each well to cover the matrix, and the plate was incubated in standard conditions, $5 \% \mathrm{CO}_{2^{-}} 95 \%$ air, at $37^{\circ} \mathrm{C}$, for $72 \mathrm{~h}$.

For histological evaluation, the cell-matrix constructs were fixed in $4 \%(\mathrm{v} / \mathrm{v})$ paraformaldehyde and embedded in paraffin. Transverse sections of $6 \mu \mathrm{m}$ were stained with hematoxylin-eosin (H\&E).

For ultrastructural observation, the constructs were fixed in $2 \%(\mathrm{v} / \mathrm{v})$ glutaraldehyde in $\mathrm{PBS}, \mathrm{pH} 7.4$, at $4^{\circ} \mathrm{C}$, for $2 \mathrm{~h}$, dehydrated in serial concentrations of ethanol, transverse cut, and mounted and visualized in the low vacuum mode at a FEI Quanta 400 ESEM (Philips, The Netherlands), at a voltage of $25 \mathrm{kV}$.

2.7. In Vitro Diffusion Test. In vitro diffusion of the entrapped CS through the dialysis membrane was assessed as previously described [26]. Briefly, the determination was comparatively carried out by placing a known quantity of L-CS suspension and equivalent amount of Col-L-CS into separate dialysis tubes (molecular weight cut-off 12,000 Da), sealed at both ends. They were placed in test tubes containing $10 \mathrm{~mL}$ PBS, $\mathrm{pH} 7.4$, and were gently shaken at $60 \mathrm{rpm}$, in a thermostated water bath at $35^{\circ} \mathrm{C}$, for $72 \mathrm{~h}$. Aliquots of $300 \mu \mathrm{L}$ solution were withdrawn, at different intervals between 0 and $72 \mathrm{~h}$, and replaced with fresh PBS. The amount of CS released from liposomal formulations was determined using Blyscan kit. Three separate experiments were conducted and the results were reported as mean $\pm \mathrm{SD}$.

2.8. Statistical Analysis. Data were expressed as mean value \pm SD for three independent samples $(n=3)$. Statistical analysis of the data was performed using the one-tailed paired Student's $t$-test, on each pair of interest. Differences were considered statistically significant at $P<0.05$, as a minimal level of significance.

\section{Results and Discussion}

3.1. Preparation and Structural Characterization of Col-L-CS. In the first stage, we prepared liposomal vesicles encapsulating CS and carried out DLS measurements of their size and polydispersity. The results for size distribution in the population of L-CS indicated a mean diameter of $523.83 \mathrm{~nm}$ \pm 10.2 , for a PI value of 0.40 . CS encapsulation efficiency in the lipid vesicles was high $(68.2 \% \pm 5.6)$, probably due to an ionic attraction between $\mathrm{O}$-sulfate groups of encapsulated CS and the amino end of the cationic lipid SA present in the lipid bilayer.

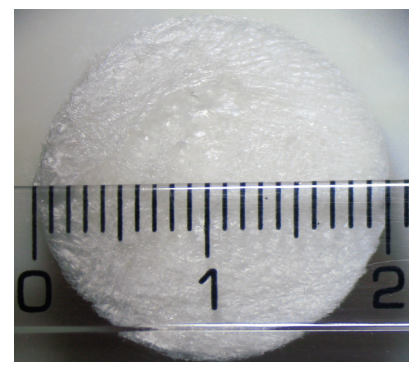

(a)

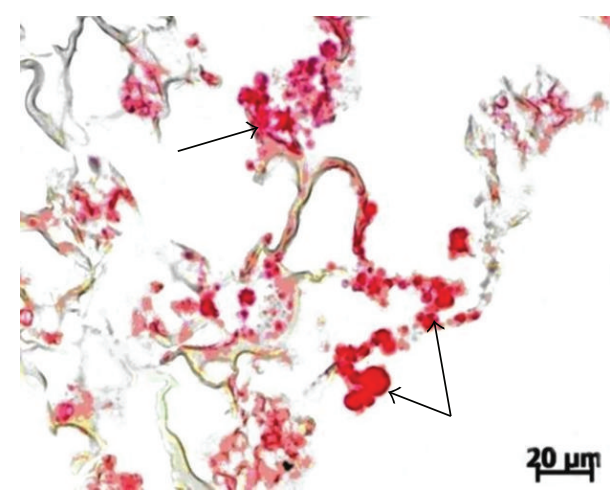

(c)

Figure 1: Top (a) and side (b) views of Col-L-CS matrix delivery system. Light micrograph of COL-L-CS matrix delivery system showing the distribution of L-CS (arrows) in the polymeric matrix (c) (Oil Red O staining).

Col type I was obtained using an enzymatic method to remove the nonhelical ends (telopeptides) of the triple helix region to result in a nonimmunogenic polymer of $308 \mathrm{kDa}$, similar to the molecular weight of tropocollagen $(300 \mathrm{kDa})$, as we previously showed [21].

Col-L-CS matrix delivery system obtained by freezedrying a mixture of Col type I gel and L-CS suspension had the macroscopic appearance presented in Figures 1(a) and $1(\mathrm{~b})$. The distribution of L-CS vesicles within the 3D porous matrix was histochemically investigated using the lipid specific Oil Red O staining (Figure 1(c)). The micrographs showed that L-CS occurred separately or in groups along COL fibrils.

The ultrastructure of the surface (Figure 2(a)) and transverse cross-section (Figure 2(b)) of COL-L-CS observed by SEM presented an alveolar network of COL fibrils with heterogeneous sized pores, compared to the honeycomb appearance of Col matrix (Figure 2(c)). Varying amounts of L-CS white deposits decorated in a greater extent the matrix surface than its center (Figures 2(a) and 2(b)).

Previous reports using TEM imaging by freeze-fracture sample preparation revealed L entrapped in a COL gel; at high magnification, Col fibrils attached to the lipid bilayer of the vesicles were observed [27]. Many studies investigated the effect of Col type I solution on L with different composition (PC, Chol, or lecithin), charge (neutral, positive, or negative), type (SUV or MLV), and their complex interaction. Biophysical studies on Col-L interaction demonstrated that Col could 


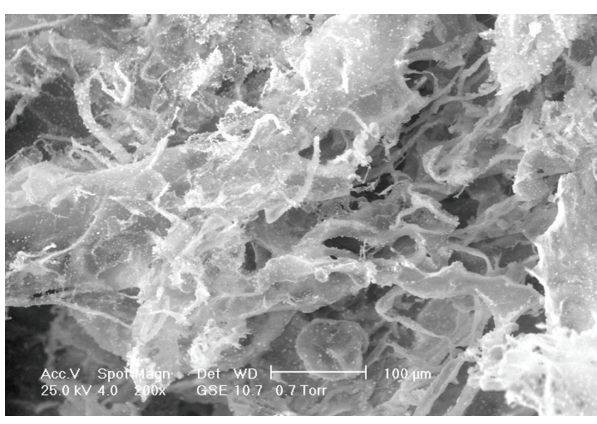

(a)

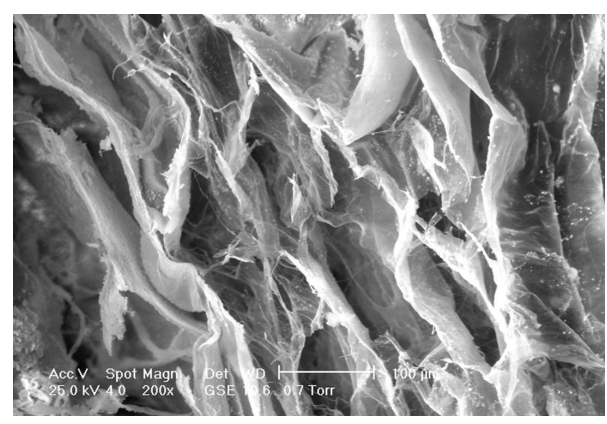

(b)

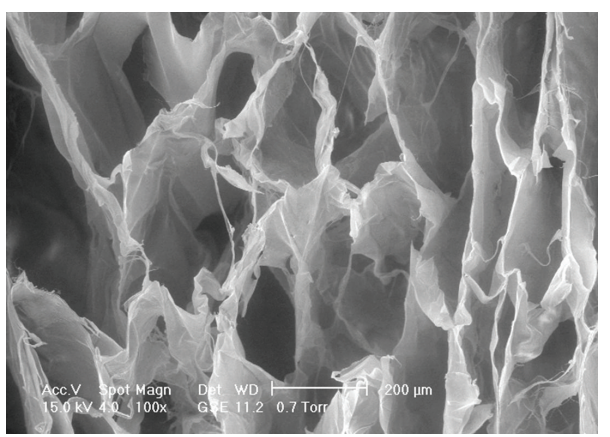

(c)

FIGURE 2: SEM micrographs showing a high amount of L-CS (white dots) distributed on Col-L-CS matrix surface (a) and L-CS (white dots) sparsely disposed on inner COL fibrils of Col-L-CS matrix transverse section (b). SEM micrograph of Col matrix transverse section ultrastructure (c).

TABLE 1: The porosity, density, mean pore size, and biodegradability of Col-L-CS matrix delivery system compared to Col matrix. Data were calculated as mean $\pm \mathrm{SD}(n=3)$.

\begin{tabular}{|c|c|c|c|c|c|}
\hline \multirow{2}{*}{ Sample } & \multirow{2}{*}{ Porosity (\%) } & \multirow{2}{*}{ Density $\left(\mathrm{g} / \mathrm{cm}^{3}\right)$} & \multirow{2}{*}{ Mean pore size $(\mu \mathrm{m})$} & \multicolumn{2}{|c|}{ Biodegradability (\%) } \\
\hline & & & & Buffer & Collagenase \\
\hline Col-L-CS & $93.22 \pm 7.41$ & $1.31 \pm 0.11$ & $231.41 \pm 35.53^{*}$ & $14.56 \pm 3.12^{*}$ & $34.95 \pm 3.91^{*}$ \\
\hline Col (control) & $97.53 \pm 8.72$ & $1.17 \pm 0.09$ & $137.52 \pm 30.92$ & $24.51 \pm 5.07$ & $46.75 \pm 4.36$ \\
\hline
\end{tabular}

${ }^{*} P<0.05$, compared to Col control matrix.

either form a protein layer at the liposomal surface, or it could be incorporated within the lipid bilayer via its hydrophobic residues [28]. It was also demonstrated that Col coating of lipid vesicles improved their chemical stability, had no effect on their fluidity, and decreased their permeability, due to electrostatic forces between positive charges of $\mathrm{Col}$ and the phospholipids from charged liposomes [29-31].

The porosity and density of Col-L-CS matrix are presented in Table 1 . The calculated values $\left(93.2 \%\right.$ and $1.31 \mathrm{~g} / \mathrm{cm}^{3}$, resp.) were similar to those of COL matrix, indicating that LCS were tightly attached to Col fibrils, without modifying the porosity of the network. The microporous structure of ColL-CS had uneven pore sizes, varying between 50 and $255 \mu \mathrm{m}$. The mean pore size calculated for Col-L-CS $(231.41 \mu \mathrm{m})$ was significantly higher $(P<0.05)$ compared to that of Col matrix $(137.52 \mu \mathrm{m})$ (Table 1$)$.

The porosity and pore size of 3D matrices are important structural characteristics for their medical use as temporary scaffolds. The porosity of a collagenic matrix could be affected by the extraction parameters, such as solvent/enzyme type and $\mathrm{pH}$, on one side, and by the processing parameters, such as freezing temperature during lyophilization, on the other side [32]. Previous studies showed that the final pore size of a lyophilized matrix could be controlled by varying the freezing temperature in the range of $-20^{\circ} \mathrm{C}$ to $-196^{\circ} \mathrm{C}$; namely, the lower the temperature, the smaller the pore size [33]. Recent reviews reported that polymeric scaffolds with pore size range between 50 and $200 \mu \mathrm{m}$ provided a favorable cell infiltration, adhesion, and proliferation, initiating new extracellular matrix secretion at the damaged site [34]. At the same time, they could also facilitate the release of loaded biofactors [35]. In our study, Col-L-CS matrix had a high value of porosity (>90\%) and mean pore size around $200 \mu \mathrm{m}$, which could favor tissue regeneration.

3.2. Biodegradability of Col-L-CS Matrix. The biodegradability is an important parameter for characterizing a medical device intended for transport and release of bioactive molecules. In our study, the results of biodegradability test in TES buffer showed that Col-L-CS matrix was stable (14.56\%), 


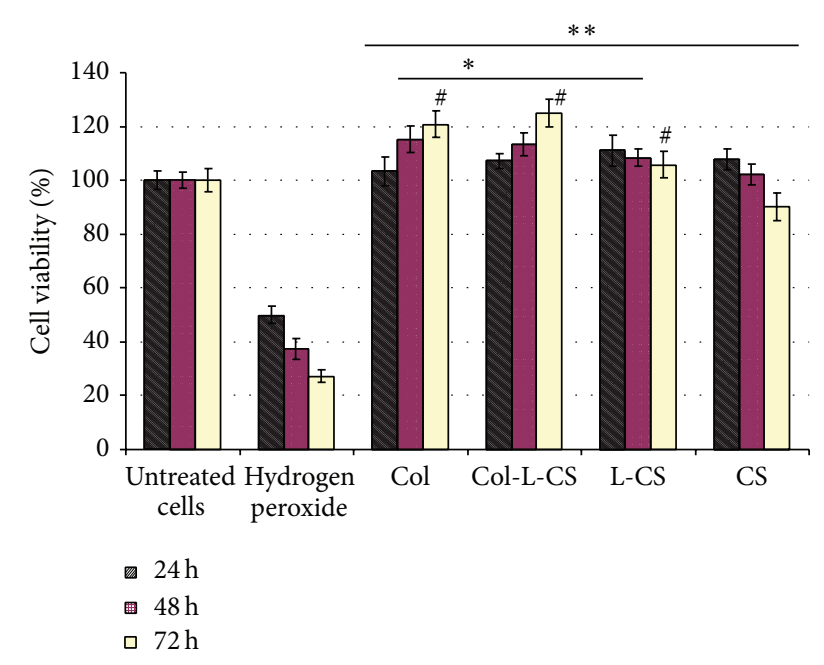

FIGURE 3: Cell viability of L929 fibroblasts cultured in the presence of Col-L-CS matrix, L-CS, Col, and CS, assessed by MTT assay, at 24, 48 , and $72 \mathrm{~h}$ of cultivation. Untreated cells in complete medium and cells cultivated in the presence of $50 \mu \mathrm{M} \mathrm{H}_{2} \mathrm{O}_{2}$ were used as controls. ${ }^{*} \mathrm{P}<0.05$, compared to untreated cells; ${ }^{*}{ }^{*} P<0.05$, compared to $\mathrm{H}_{2} \mathrm{O}_{2}$ group; ${ }^{\#} \mathrm{P}<0.05$, compared to CS.

while in the presence of collagenase, the matrix was partially degraded (34.95\%), after $6 \mathrm{~h}$ of incubation (Table 1). It was observed that Col-L-CS was significantly less degraded $(P<$ 0.05) than Col control, using in situ mimicking conditions. The results indicated a good candidate for both controlled CS release and cell infiltration into the matrix. It is possible that the attachment and interaction between $\mathrm{L}$ and Col could increase $\mathrm{Col}$ resistance to solubilization by partially hindering the site of collagenase action, namely, the amino bond of glycine in the specific sequence X-Gly-Pro. Other porous matrices based on Col showed their metabolic stability and active role in articular cartilage tissue engineering [36].

3.3. In Vitro Cytotoxicity of Col-L-CS. According to the International Standard ISO 10993-5, there are several specific methods to analyze the cytotoxicity of medical devices, which involve different aspects of cell function, like cell viability and proliferation, loss of membrane integrity, cell adhesion, and morphology. In our study, we used two in vitro models, one for indirect cytotoxicity testing by cultivation of Col-L-CS samples in the culture medium of L929 cells and one for direct cytotoxicity testing by cell cultivation after injection in ColL-CS matrix, for $72 \mathrm{~h}$. In the first model, we analyzed the cells for viability by MTT assay and morphology by H\&E staining. In the second model, we analyzed the cell-matrix construct by light and electron microscopy.

3.3.1. Indirect Cytotoxicity. Cell viability was quantified by MTT test, indicator of living cells metabolism, in particular, of mitochondrial enzymes activity. We compared the cytotoxicity of Col-L-CS matrix to that of L-CS, Col alone, and free CS cultivated in the same conditions. MTT results presented in Figure 3 showed that the percentage of viable cells varied between $124.82 \%$ in Col-L-CS group and $90.16 \%$ in CS group, after $72 \mathrm{~h}$ of cultivation, indicating that all tested samples were not cytotoxic, according to ISO 10993-5. Moreover, the values were significantly higher $(P<0.05)$ than in untreated cells (negative control), excepting CS group, Col matrix at $24 \mathrm{~h}$, and L-CS at $72 \mathrm{~h}$. Statistic examination of the $72 \mathrm{~h}$ records for each sample-control pair showed that the viability of L929 fibroblasts was similar in Col-L-CS group to that of Col group (no statistical significance, $P>0.05$ ). The values were significantly higher $(P<0.05)$ in Col-L-CS group than in CS group, at $72 \mathrm{~h}$ of cultivation. L-CS group had also significantly higher $(P<0.05)$ values of cell viability compared to CS group, at $72 \mathrm{~h}$ of cultivation, but significantly lower $(P<$ $0.05)$ than Col-L-CS group. These results indicated that ColL-CS matrix had the ability to stimulate L929 fibroblast cell metabolism.

Cell morphology was observed at the membrane, cytoplasm, and nucleus level after specific staining. Representative light micrographs of cells cultivated in the presence of studied samples (Col-L-CS, Col, L-CS, and CS) for $72 \mathrm{~h}$ are shown in Figure 4. The matrices floated in the culture medium without contact with the adhered cell layer. Cells cultivated in the presence of Col-L-CS matrix showed the same morphology as untreated cells (control), with a spindle shape morphology, clear cytoplasm, and euchromatic nuclei with 1-2 nucleoli. The cell density reached an almost complete monolayer after $72 \mathrm{~h}$ of cultivation. Also, fibroblast cells cultivated with Col matrix maintained their specific morphology. In the presence of L-CS and CS solutions, cells with normal morphology were observed, but with a slightly lower density than that of Col-L-CS treated cells.

There was a good correlation between the morphological observations and the quantitative data of cell viability. Both methods indicated that Col-L-CS was a biocompatible matrix that could stimulate cell growth and metabolism in a higher degree than the therapeutic agent CS.

3.3.2. Direct Cytotoxicity. The effect of Col-L-CS matrix on the viability and distribution of cells injected and cultivated in direct contact was analyzed by SEM and light microscopy observations. SEM micrographs showed that a high number of cell groups were distributed on the matrix surface, after $72 \mathrm{~h}$ of cultivation (Figure 5(a)). Histological staining of cell-matrix construct showed that the cells clustered into the pores and presented normal morphology (Figure 5(b)). Moreover, the cells were located along Col fibrils. All these microscopy observations indicated that Col-L-CS porous matrix promoted cell encapsulation and clustering within its alveolar structure.

Previous studies reported that a Col type I gel was very efficient in filling small cartilage defects, either as cellfree matrix or cell carrier and Col-based matrices which promoted in vitro migration and proliferation of chondrocytes [37]. Col-GAG lyophilized scaffolds were fabricated for bone tissue repair, and certain pore sizes induced osteogenic behavior of different cell types [32]. Also, Col-GAG scaffolds seeded with keratinocytes led to regeneration of both dermis and epidermis [34]. Col is known as a matricellular protein 

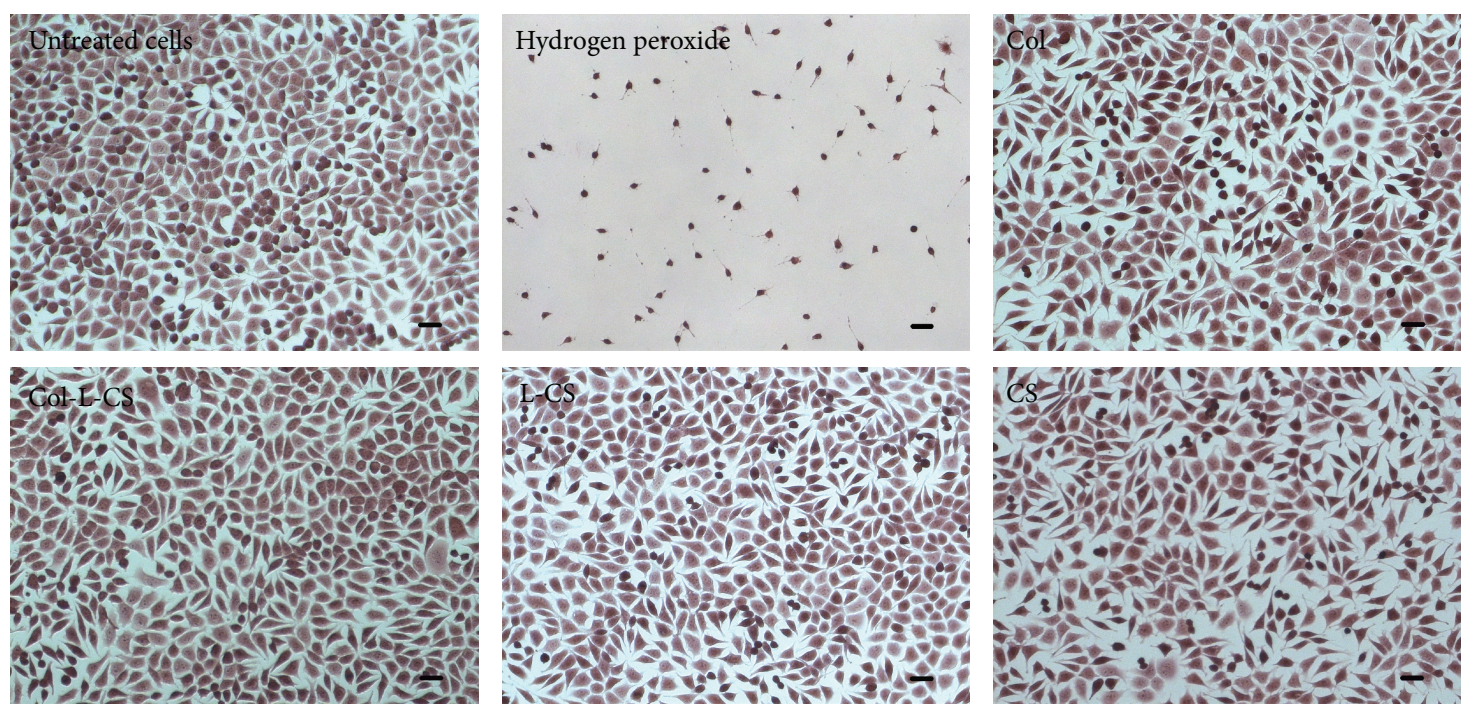

FIGURE 4: Light micrographs of L929 cells cultivated with Col-L-CS and Col matrices and L-CS and CS solutions, in standard conditions, for $72 \mathrm{~h}$, showing specific fibroblast morphology and a good cytocompatibility of the samples. Untreated cells and cells treated with $50 \mu \mathrm{M} \mathrm{H}_{2} \mathrm{O}_{2}$ were used as controls (H\&E staining) (bar $=10 \mu \mathrm{m})$.

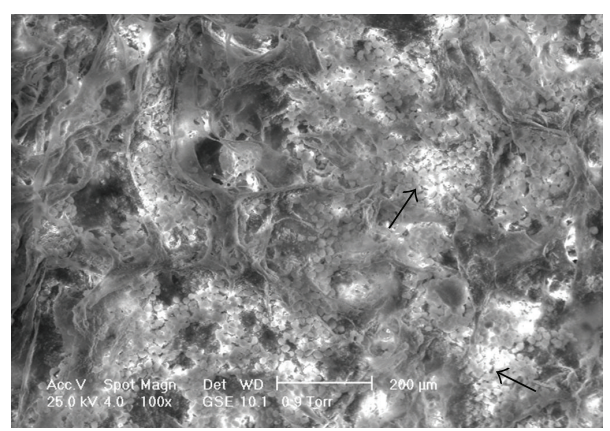

(a)

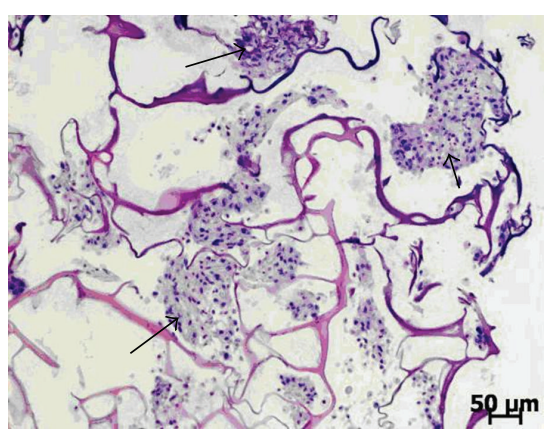

(b)

FIGURE 5: SEM micrograph presenting a high number of cell groups (arrows) on Col-L-CS matrix surface, after $72 \mathrm{~h}$ of cultivation (a). Histological observation of Col-L-CS matrix showing cell colonization (arrows) within the pores, after $72 \mathrm{~h}$ of cultivation (b) (H\&E staining).

acting as cellular signaling molecule via ligands in the form of Arg-Gly-Asp (RGD) binding sequences [38]. In our study, the enzymatically prepared Col type I preserved its triple helical conformation that it is known to promote integrin-mediated cell adhesion, proliferation, and differentiation [39]. Based on its structural characteristics and functional properties, we consider that this Col-L-CS hydrophilic matrix might be a useful scaffold for initiating tissue regeneration.

3.4. In Vitro CS Release. The profile of CS release from ColL-CS matrix delivery system was studied in physiological

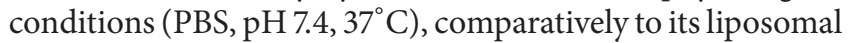
formulation, L-CS, as presented in Figure 6. Both samples gradually released CS, but not to a constant rate. In the first $360 \mathrm{~min}$ (stage I), CS release profiles had the highest slope, while a smaller profile slope was observed in the second stage between 360 and $1800 \mathrm{~min}$ (II). The third stage (III) almost reached a plateau and presented the highest difference between the two profiles. The data showed that Col-L-CS matrix system presented a significantly lower $(P<$ 0.05 ) quantity of CS released than L-CS, after $4320 \mathrm{~min}$ of incubation. After the first day of incubation, only $33 \%$ CS was released by the matrix delivery system, compared to $57 \%$ CS released by the liposomal formulation. At the end of the incubation period, 55\% CS was released from Col-LCS matrix and 93\% CS was released from L-CS formulation. These results indicated that embedment of L-CS within Col matrix significantly decreased $(P<0.05)$ the released CS quantity, even after $120 \mathrm{~min}$ of incubation $(1.76 \%$ from ColL-CS and $12.14 \%$ from L-CS).

Previous studies showed that drug liposomal formulations provided greater solubility, longer circulation times, focused delivery, and better therapeutic index, compared to drug free form [40]. Our observations on Col-L-CS matrix delivery system supported the reports on interaction between the lipid bilayer and Col fibrils that could offer both a better stability and a lower permeability of L $[30,31]$. On the 


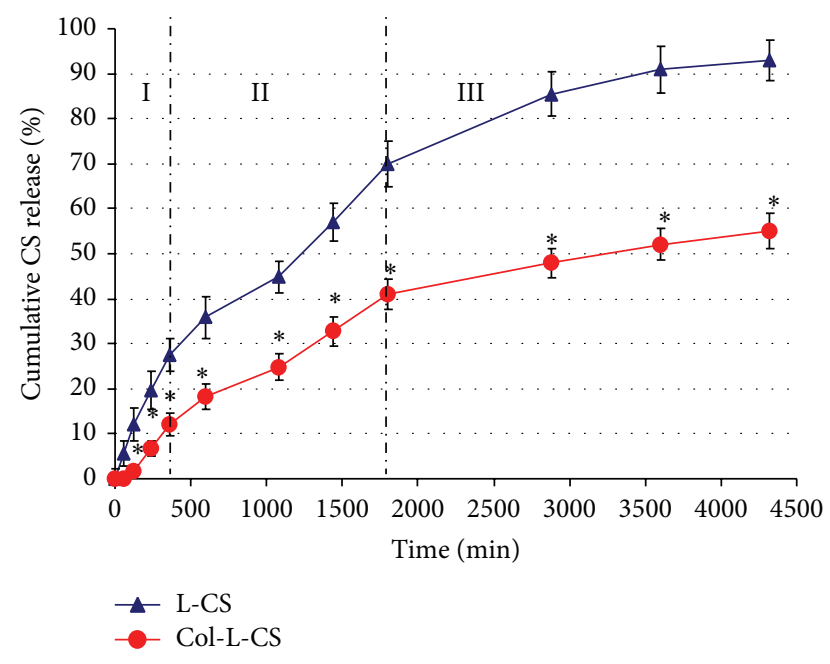

FIGURE 6: The profile of CS cumulative release from Col-L-CS and LCS delivery systems, in physiological conditions, at predetermined periods showed three stages (I-III). Data are expressed as mean \pm $\mathrm{SD}(n=3) .{ }^{*} P<0.05$, compared to L-CS.

other side, the structural parameters of the matrix (porosity, biodegradability) could further modulate the drug release process. These factors and their complex interrelation might provide a better controlled release of drug from Col-L-CS matrix, compared to L-CS. The proposed DDS improved CS diffusion rate and, thus, could lead to a sustained delivery at the injured site and prolongation of its action duration. In turn, orally administered CS analysis over $48 \mathrm{~h}$ showed a maximum drug concentration $(22 \%)$ in the first $2-6 \mathrm{~h}$, indicating poor drug bioavailability at the injured site [41].

\section{Conclusion}

A 3D porous matrix of Col embedding the liposomal formulation of CS was prepared by freeze-drying and was physicochemically and biologically characterized. Its morphological appearance was similar to that of Col scaffold, presenting a network of interconnected Col fibrils unevenly covered with white deposits of L-CS. The porosity and density were similar to those of Col control scaffold, but its biodegradability was significantly lower $(P<0.05)$. In vitro cytotoxicity tests highlighted Col-L-CS advantage over CS alone, namely, a significantly higher $(P<0.05)$ cytocompatibility in L929 cell culture and cell metabolism stimulation. Due to cell encapsulation facility, Col-L-CS might also exert a potential regenerative action at the site of inflammation, unlike L-CS delivery system. CS release was better controlled from ColL-CS matrix, compared to the liposomal formulation, L-CS. In conclusion, Col-L-CS matrix system reported here could have potential use in local delivery of therapeutic agents. Furthermore, investigations are required to confirm its in vitro and in vivo anti-inflammatory and regenerative capacity.

\section{Conflict of Interests}

The authors declare that there is no conflict of interests regarding the publication of this paper.

\section{Acknowledgments}

The authors thank Professor Dr. D. Bojin from BIOMAT Center, Bucharest, for helping with SEM images acquisition. This work was supported by the Romanian Project BIODIV 102 and the Romanian Academy Project no. 3/2013.

\section{References}

[1] M. Goldberg, R. Langer, and X. Jia, "Nanostructured materials for applications in drug delivery and tissue engineering," Journal of Biomaterials Science, Polymer Edition, vol. 18, no. 3, pp. 241-268, 2007.

[2] T. M. Allen and P. R. Cullis, "Liposomal drug delivery systems: from concept to clinical applications," Advanced Drug Delivery Reviews, vol. 65, no. 1, pp. 36-48, 2013.

[3] J. M. Metselaar and G. Storm, "Liposomes in the treatment of inflammatory disorders," Expert Opinion on Drug Delivery, vol. 2, no. 3, pp. 465-476, 2005.

[4] Y. Barenholz, C. Bombelli, M. G. Bonicelli et al., "Influence of lipid composition on the thermotropic behavior and size distribution of mixed cationic liposomes," Journal of Colloid and Interface Science, vol. 356, no. 1, pp. 46-53, 2011.

[5] A. S. Vanniasinghe, V. Bender, and N. Manolios, "The potential of liposomal drug delivery for the treatment of inflammatory arthritis," Seminars in Arthritis and Rheumatism, vol. 39, no. 3, pp. 182-196, 2009.

[6] M. Trif, A. Roseanu, J. H. Brock, and J. M. Brewer, “Designing lipid nanostructures for local delivery of biologically active macromolecules," Journal of Liposome Research, vol. 17, no. 3-4, pp. 237-248, 2007.

[7] J. F. Coelho, P. C. Ferreira, P. Alves et al., "Drug delivery systems: advanced technologies potentially applicable in personalized treatments," The EPMA Journal, vol. 1, no. 1, pp. 164-209, 2010.

[8] M. Peran, M. A. Garcia, E. Lopez-Ruiz et al., "Functionalized nanostructures with application in regenerative medicine," International Journal of Molecular Sciences, vol. 13, no. 3, pp. 3847-3886, 2012.

[9] N. Ashammakhi, R. L. Reis, and F. Chiellini, Eds., Topics in Tissue Engineering, E-Book, 1st edition, 2008.

[10] A. Guimaires, A. Martins, E. D. Pinho, S. Faria, R. L. Reis, and N. M. Neves, "Solving cell infiltration limitations of electrospun nanofiber meshes for tissue engineering applications," Nanomedicine, vol. 5, no. 4, pp. 539-554, 2010.

[11] S. L. Montes-Fonseca, E. Orrantia-Borunda, A. Duarte-Moller et al., "Cytotoxicity of carbon nanotubes on J774 macrophages is a purification-dependent effect," Journal of Nanomaterials, vol. 2012, Article ID 715838, 7 pages, 2012.

[12] Y. Sharma, A. Tiwari, S. Hattori et al., "Fabrication of conducting electrospun nanofibers scaffold for three-dimensional cells culture," International Journal of Biological Macromolecules, vol. 51, no. 4, pp. 627-631, 2012.

[13] P. B. Malafaya, G. A. Silva, and R. L. Reis, "Natural-origin polymers as carriers and scaffolds for biomolecules and cell delivery in tissue engineering applications," Advanced Drug Delivery Reviews, vol. 59, no. 4-5, pp. 207-233, 2007. 
[14] C. Peptu, M. Popa, and S. G. Antimisiaris, "Release of liposomeencapsulated calcein from liposome entrapping gelatincarboxymethylcellulose films: a presentation of different possibilities," Journal of Nanoscience and Nanotechnology, vol. 8, no. 5, pp. 2249-2258, 2008.

[15] J. D. Kretlow, L. Klouda, and A. G. Mikos, "Injectable matrices and scaffolds for drug delivery in tissue engineering," Advanced Drug Delivery Reviews, vol. 59, no. 4-5, pp. 263-273, 2007.

[16] K. L. Miller and D. O. Clegg, "Glucosamine and chondroitin sulfate," Rheumatic Disease Clinics of North America, vol. 37, no. 1, pp. 103-118, 2011.

[17] O. Craciunescu, L. Moldovan, M. Moisei, and M. Trif, "Liposomal formulation of chondroitin sulphate enhances its antioxidant and anti-inflammatory potential in L929 fibroblast cell line," Journal of Liposome Research, vol. 23, no. 2, pp. 145-153, 2013.

[18] M. R. Mozafari, "Nanoliposomes: preparation and analysis," Methods in Molecular Biology, vol. 605, pp. 29-50, 2010.

[19] J. Wang, Y. Yuan, C. Liu, D. Zhu, X. Shen, and B. Yang, "Preparation and pharmaceutical/pharmacodynamic evaluation of topical brucine-loaded liposomal hydrogel," Journal of Materials Science: Materials in Medicine, vol. 20, no. 10, pp. 2075-2084, 2009.

[20] M. M. French, S. Rose, J. Canseco, and K. A. Athanasiou, "Chondrogenic differentiation of adult dermal fibroblasts," Annals of Biomedical Engineering, vol. 32, no. 1, pp. 50-56, 2004.

[21] L. Moldovan, E. I. Oprita, O. Craciunescu, C. Tardei, D. Bojin, and O. Zarnescu, "Histochemical and scanning electron microscopic characterization of tricalcium phosphate-collagen conjugated sponges," Romanian Biotechnological Letters, vol. 9, no. 6, pp. 1887-1893, 2004.

[22] J. Cuppoletti, Ed., Nanocomposites and Polymers with Analytical Methods, InTech, Rijeka, Croatia, 1st edition, 2011.

[23] S. M. Zhang, F. Z. Cui, S. S. Liao, Y. Zhu, and L. Han, "Synthesis and biocompatibility of porous nano-hydroxyapatite/collagen/alginate composite," Journal of Materials Science: Materials in Medicine, vol. 14, no. 7, pp. 641-645, 2003.

[24] A. Atala and R. P. Lanza, Eds., Methods of Tissue Engineering, Academic Press, Orlando, Fla, USA, 1st edition, 2002.

[25] T. Mosmann, "Rapid colorimetric assay for cellular growth and survival: application to proliferation and cytotoxicity assays," Journal of Immunological Methods, vol. 65, no. 1-2, pp. 55-63, 1983.

[26] N. K. Jain and S. K. Jain, "Development and in vitro characterization of galactosylated low molecular weight chitosan nanoparticles bearing doxorubicin," AAPS PharmSciTech, vol. 11, no. 2, pp. 686-697, 2010.

[27] A. W. Pederson, J. W. Ruberti, and P. B. Messersmith, "Thermal assembly of a biomimetic mineral/collagen composite," Biomaterials, vol. 24, no. 26, pp. 4881-4890, 2003.

[28] M. M. Mady, "Biophysical studies on collagen-lipid interaction," Journal of Bioscience and Bioengineering, vol. 104, no. 2, pp. 144$148,2007$.

[29] X. Shi, W. Ma, C. Sun, and S. Wu, "The aggregation behavior of collagen in aqueous solution and its property of stabilizing liposomes in vitro," Biomaterials, vol. 22, no. 12, pp. 1627-1634, 2001.

[30] X.-Y. Shi, C.-M. Sun, and S.-K. Wu, "Evaluation of in vitro stability of small unilamellar vesicles coated with collagen and chitosan," Polymer International, vol. 48, no. 3, pp. 212-216, 1999.
[31] M. Pajean and D. Herbage, "Effect of collagen on liposome permeability," International Journal of Pharmaceutics, vol. 91, no. 2-3, pp. 209-216, 1993.

[32] C. M. Murphy and F. J. O'Brien, "Understanding the effect of mean pore size on cell activity in collagen-glycosaminoglycan scaffolds," Cell Adhesion \& Migration, vol. 4, no. 3, pp. 377-381, 2010.

[33] V. Karageorgiou and D. Kaplan, "Porosity of 3D biomaterial scaffolds and osteogenesis," Biomaterials, vol. 26, no. 27, pp. 5474-5491, 2005.

[34] I. V. Yannas, D. S. Tzeranis, B. A. Harley, and P. T. So, "Biologically active collagen-based scaffolds: advances in processing and characterization," Philosophical Transactions of the Royal Society A: Mathematical, Physical and Engineering Sciences, vol. 368, no. 1917, pp. 2123-2139, 2010.

[35] Q. L. Loh and C. Choong, "Three-dimensional scaffolds for tissue engineering applications: role of porosity and pore size," Tissue Engineering B, vol. 19, no. 6, pp. 485-502, 2013.

[36] G. Bock and J. Goode, Eds., Tissue Engineering of Cartilage and Bone, John Wiley \& Sons, Chichester, UK, 1st edition, 2003.

[37] T. Efe, C. Theisen, S. Fuchs-Winkelmann et al., "Cell-free collagen type I matrix for repair of cartilage defects- clinical and magnetic resonance imaging results," Knee Surgery, Sports Traumatology, Arthroscopy, vol. 20, no. 10, pp. 1915-1922, 2012.

[38] M. Goldberg, S. Lacerda-Pinheiro, F. Priam et al., "Matricellular molecules and odontoblast progenitors as tools for dentin repair and regeneration," Clinical Oral Investigations, vol. 12, no. 2, pp. 109-112, 2008.

[39] Z. Ruszczak, "Effect of collagen matrices on dermal wound healing," Advanced Drug Delivery Reviews, vol. 55, no. 12, pp. 1595-1611, 2003.

[40] D. Bitounis, R. Fanciullino, A. Iliadis, and J. Ciccolini, "Optimizing druggability through liposomal formulations: new approaches to an old concept," ISRN Pharmaceutics, vol. 2012, Article ID 738432, 11 pages, 2012.

[41] N. Volpi, "Oral bioavailability of chondroitin sulfate (Condrosulf) and its constituents in healthy male volunteers," Osteoarthritis and Cartilage, vol. 10, no. 10, pp. 768-777, 2002. 

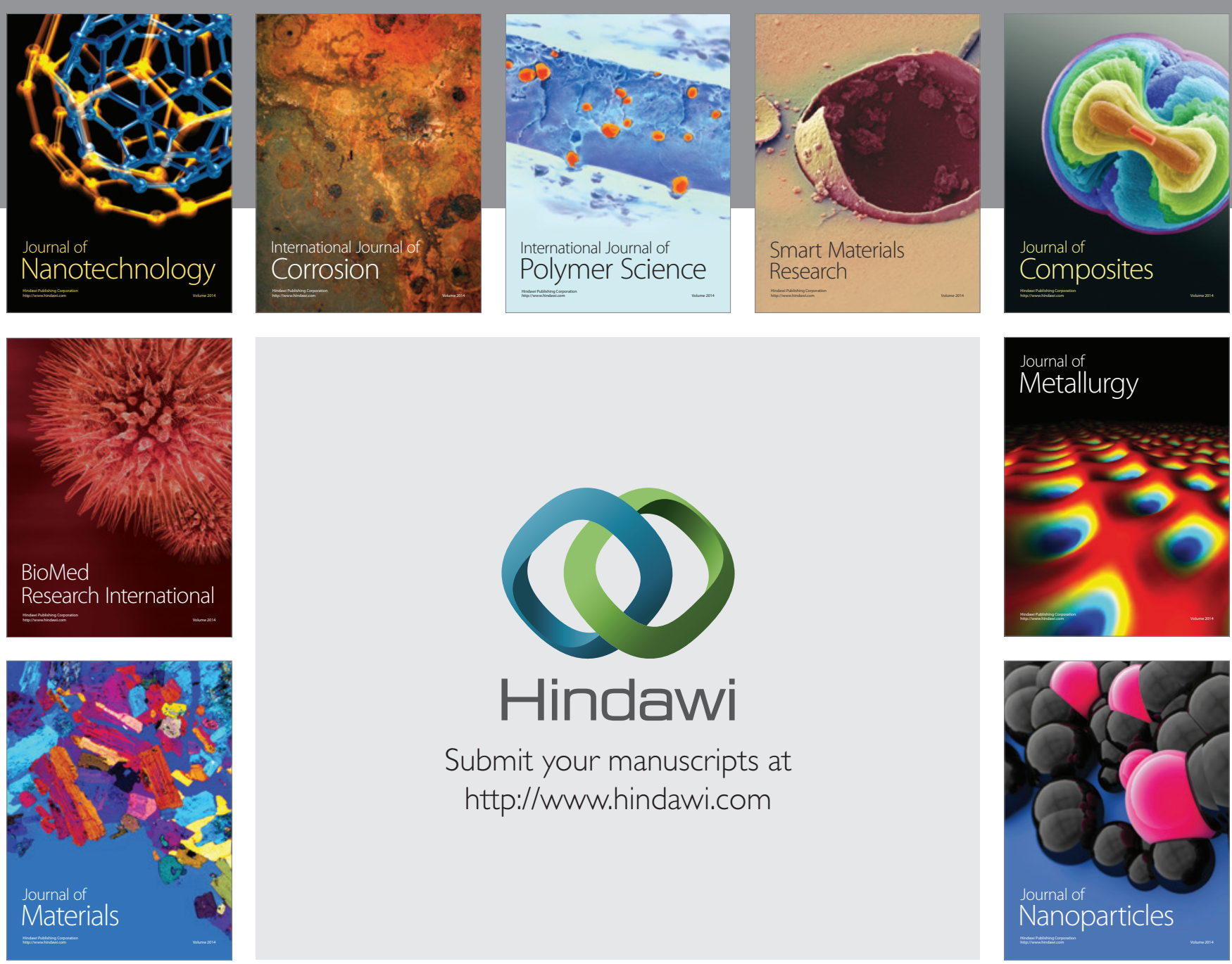

Submit your manuscripts at http://www.hindawi.com
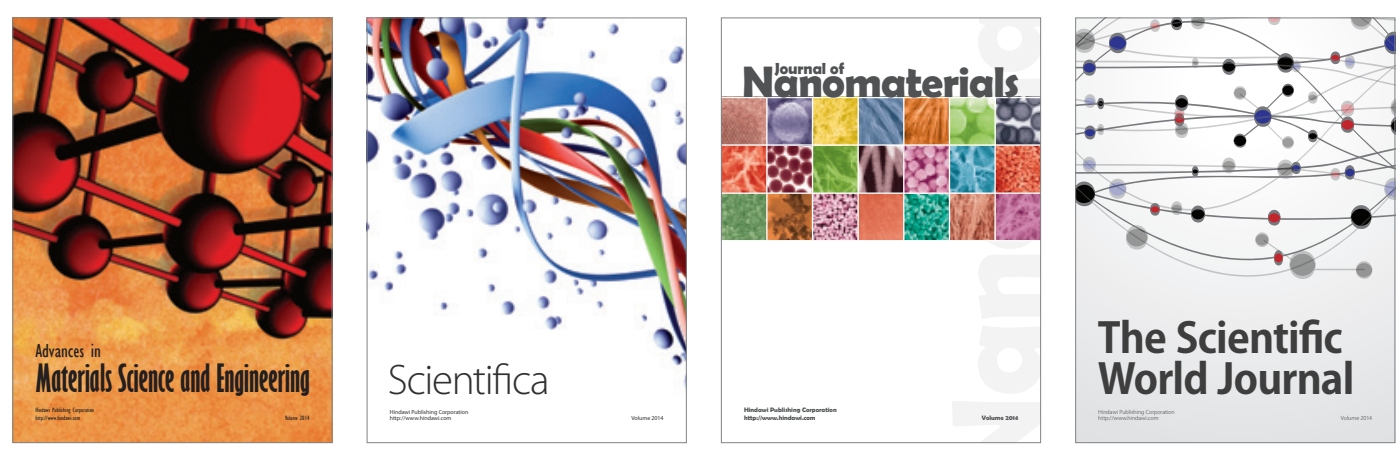

\section{The Scientific World Journal}
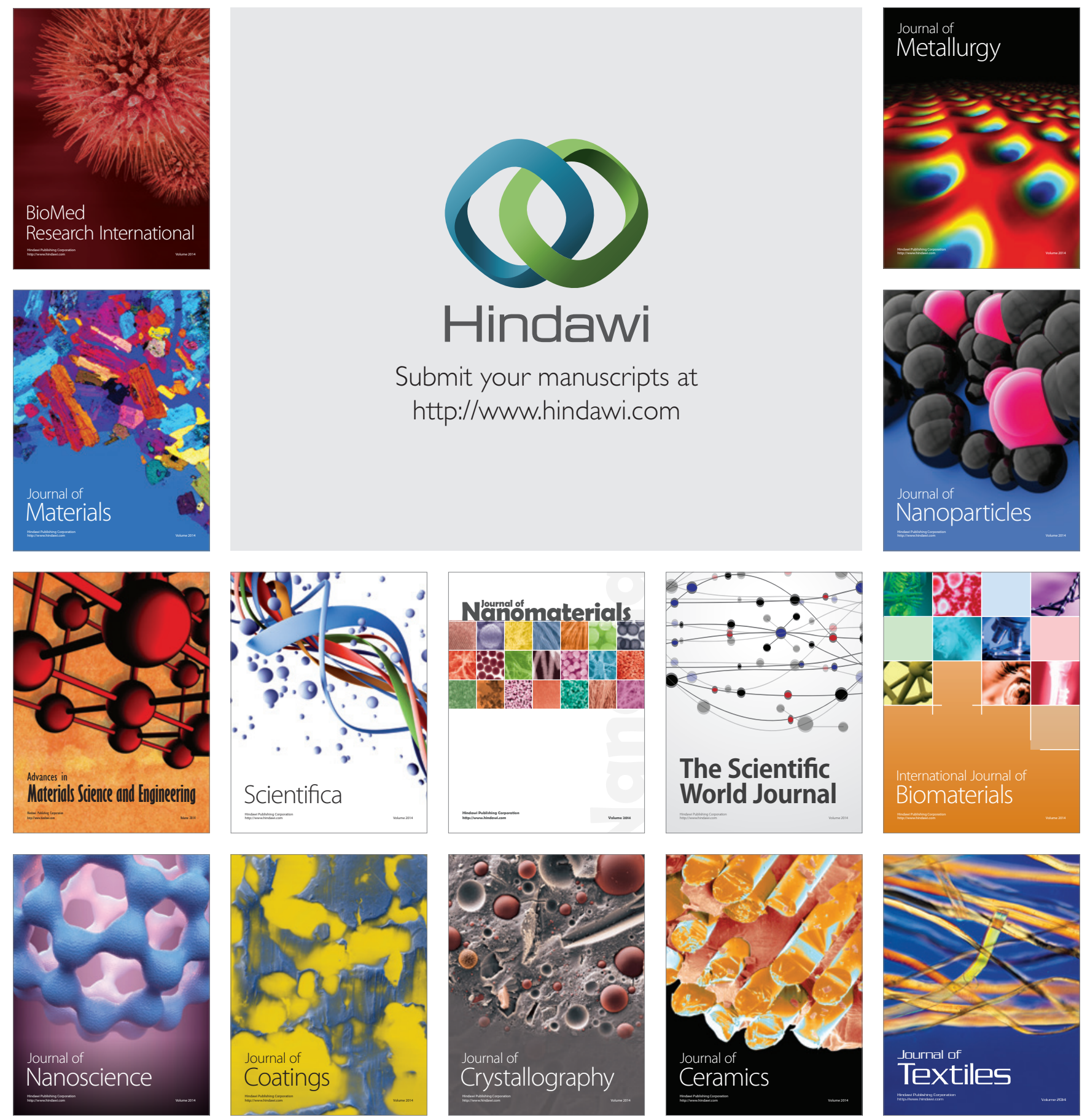